\title{
A web service based tool to plan atmospheric research flights
}

\author{
M. Rautenhaus, G. Bauer, and A. Dörnbrack \\ Deutsches Zentrum für Luft- und Raumfahrt, Institut für Physik der Atmosphäre, Oberpfaffenhofen, Germany \\ Correspondence to: M. Rautenhaus (marc.rautenhaus@dlr.de)
}

Received: 10 August 2011 - Published in Geosci. Model Dev. Discuss.: 1 September 2011

Revised: 13 January 2012 - Accepted: 13 January 2012 - Published: 17 January 2012

\begin{abstract}
We present a web service based tool for the planning of atmospheric research flights. The tool provides online access to horizontal maps and vertical cross-sections of numerical weather prediction data and in particular allows the interactive design of a flight route in direct relation to the predictions. It thereby fills a crucial gap in the set of currently available tools for using data from numerical atmospheric models for research flight planning. A distinct feature of the tool is its lightweight, web service based architecture, requiring only commodity hardware and a basic Internet connection for deployment. Access to visualisations of prediction data is achieved by using an extended version of the Open Geospatial Consortium Web Map Service (WMS) standard, a technology that has gained increased attention in meteorology in recent years. With the WMS approach, we avoid the transfer of large forecast model output datasets while enabling on-demand generated visualisations of the predictions at campaign sites with limited Internet bandwidth. Usage of the Web Map Service standard also enables access to thirdparty sources of georeferenced data. We have implemented the software using the open-source programming language Python. In the present article, we describe the architecture of the tool. As an example application, we discuss a case study research flight planned for the scenario of the 2010 Eyjafjalla volcano eruption. Usage and implementation details are provided as Supplement.
\end{abstract}

\section{Introduction}

An essential element of atmospheric research is in-situ and remote sensing observations from aircraft. For this purpose, a fleet of various aircraft targeting atmospheric regions from the boundary layer to the stratosphere is in use (e.g. Schumann, 2007). To carry out successful research flights for atmospheric measurements, thorough flight planning is as essential as the aircraft and its instruments. The focus of atmospheric research flight planning is to explore large amounts of atmospheric prediction and observation data in order to extract specific regions of interest. Subsequently, a flight route is designed considering the scientific objectives of the campaign, the predicted atmospheric situation, and instrument, aircraft and legal constraints (illustrated in Fig. 1).

Several efforts have been started in recent years to support the planning of flights during aircraft-based field campaigns. Besides the application of general purpose tools for the exploration of weather prediction and observation data such as Unidata's Integrated Data Viewer (Murray et al., 2003, 2009), tools specifically designed to assist flight planning mission scientists include NASA's Real Time Mission Monitor (Blakeslee et al., 2007) and the Waypoint Planning Tool (He et al., 2010) developed by the University of Alabama, Huntsville, and the NASA Marshall Space Flight Center. In this paper, we present an alternative approach for a lightweight, modular system that we call the "Mission Support System" (MSS). By allowing the interactive design of a flight route in direct relation to atmospheric predictions, the MSS fills a crucial gap in the set of tools available for using data from numerical atmospheric models for research flight planning. The development of the MSS was motivated by the demand at the German Aerospace Centre's Institute of Atmospheric Physics (DLR/IPA) for having a tool available for Germany's new High Altitude and Long Range (HALO) research aircraft ${ }^{1}$ that is focused on the primary needs of mission scientists responsible for planning a research flight. The MSS focusses on the following requirements: (1) interactive exploration of available atmospheric forecasts, (2) interactive flight planning in relation to these forecasts, (3) computation of expected flight performance to assess the technical feasibility (in terms of total distance and vertical profile) of a flight, (4) no transfer of large forecast data files to the campaign site to allow deployment at

\footnotetext{
${ }^{1}$ http://www.halo.dlr.de
} 


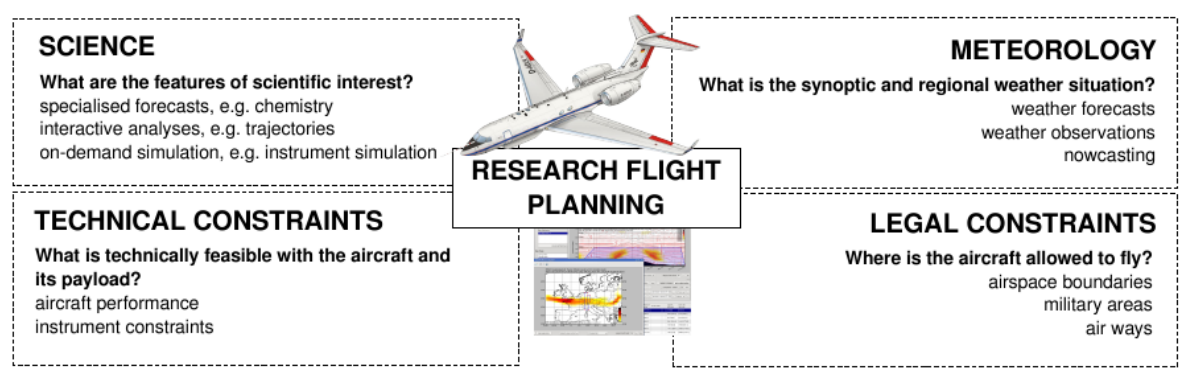

Fig. 1. The major aspects of research flight planning that have to be considered when designing a flight route.

remote locations and (5) low demand on hardware resources. Usage of open-source rapid application development tools was favoured to allow for straightforward extensibility of the program sources to adapt to future mission-specific requirements and to avoid licensing issues.

We have approached the task with a web service based software architecture. All computations requiring direct access to the output datasets of the employed forecast models (in the here presented version of the tool visualisations and flight performance computations) are separated from the user interface program. They are made accessible to the latter as services through an Internet connection, that is, the user interface program (the client) can send requests to a server on which computations on the data are carried out. Results of the computations (for instance, a forecast map) are sent back to the client. For forecast imagery, we apply the Web Map Service standard (WMS, De la Beaujardiere, 2002, 2006) defined by the Open Geospatial Consortium ${ }^{2}$ (OGC), which defines an interface to request images of maps of geospatial data through a simple HTTP-based (Hypertext Transfer Protocol) interface. For flight performance computations, a similar interface has been designed.

Although commonplace in Geographic Information Systems (GIS), the WMS standard has not been widely used in the atmospheric domain. One reason are several problems that have been encountered in encoding, for instance, time and space dimensions and in requesting vertical profiles and cross-sections. However, since an initial "Workshop on the use of OGC Web Services in Meteorology", hosted in 2008 by the European Centre for Medium Range Weather Forecasts (ECMWF) in Reading, UK, the standard is gaining increased attention in the meteorological community as a means for the standardised exchange of meteorological maps. For the MSS, we extend the standard in its version 1.1.1 to also handle vertical cross-sections. This allows us to use the WMS technology to avoid the transfer of large forecast model output datasets while enabling on-demand generated visualisations of the predictions at remote campaign sites with limited Internet bandwidth. An additional advantage is the interoperability obtained by using the standard -

\footnotetext{
${ }^{2}$ http://www.opengeospatial.org
}

as an increasing number of online geospatial data sources (including atmospheric data such as satellite and radar) are available through WMS, we readily gain access to additional data that is potentially useful for aircraft-based field campaigns.

It is the purpose of this article to present the architectural concept of the tool and to demonstrate the applicability of the software with a case study. Furthermore, our approach to using the WMS standard for atmospheric prediction data will be discussed. First, in Sect. 2, we describe the current state of the art in research flight planning and introduce available technologies including the WMS. The software architecture is described in Sect. 3, as well as our WMS extensions. An evaluation of the MSS by means of planning a flight in the scenario of the 2010 Eyjafjalla volcano eruption is presented in Sect. 4, and we conclude with a summary and an overview of in our opinion useful future research and development directions in Sect. 5. Usage and implementation details are provided as Supplement.

\section{Atmospheric research flight planning}

Aircraft-based field campaigns are often supported with a multitude of tools for different purposes. These range from specifically set up numerical models to software that enables the mission scientists to visualise and interact with the data. However, the efforts for mission support for airborne observations have received little attention in literature. From a DLR point of view, we will in the following briefly review tools that have been employed during recent field campaigns with involvement of DLR instruments (including in-situ sensors and remote sensing, e.g. lidar) and discuss software and technological trends that are of interest for mission planning.

\subsection{Challenges of planning research missions}

During field campaigns with participation of DLR's Institute of Atmospheric Physics, mission scientists in recent years had access to numerical weather prediction (NWP) data from one or more meteorological services, for instance, from the German Weather Service (DWD) or the ECMWF. Standard 
meteorological observations including satellite and radar images or radiosonde soundings were also employed. In addition, campaign-specific prediction and observational data was used. This included numerical models to predict specific atmospheric processes, for example, CLaMS (Konopka et al., 2004), FLEXPART (Stohl et al., 2005), and MATCHMPIC (Lawrence et al., 1999) for chemical forecasts, or MM5 to predict mesoscale dynamical features (Dörnbrack et al., 1998).

Observations such as ground based lidar measurements were utilised, and nowcast tools were employed to predict severe weather (Huntrieser et al., 2007). Furthermore, tools for "on-demand" predictions were commonly in use. For instance, Lagrangian trajectory and particle models (e.g. LAGRANTO, Wernli and Davies, 1997, or CLaMS or FLEXPART) provided insight into air movements and thus enabled flight manoeuvres such as self-matched flights (Schofield et al., 2008) or Lagrangian dispersion experiments (the tracking of artificial trace gases in the atmosphere). Also, predicted lidar backscatter has been used (Klanner, 2009).

Typically, a flight plan is drafted from the visual exploration of the available data and the application of on-demand tools. In this respect, prediction tools and visualisations have until now been widely independent and standalone. The following description of the POLARCAT-GRACE 2008 campaign (e.g. Roiger et al., 2011) represents an - in our experience - typical situation. During POLARCAT-GRACE 2008, output data from the ECMWF NWP model and the chemical models CLaMS and FLEXPART were visualised individually by three forecast teams. LAGRANTO and FLEXPART, which were run on demand, were stand-alone applications. Visualisations of the prediction data were available as images with pre-defined map projections, extent, and colour schemes, partly accessible through web pages. Generating new images (e.g. vertical cross-sections) was script-based and time consuming. Lacking fast Internet access on the aircraft base in Greenland, no prediction data could be transferred to the campaign site and computations and visualisations had to be carried out remotely. Also, it was impossible to overlay data from the different models within the same image, or to run both Lagrangian models with just once defining the input parameters. Once suitable measurement areas were identified from the prediction data, a flight plan was designed manually on paper. The plan with its intended flight manoeuvres was discussed with the pilots, who eventually designed the official flight route that was finally handed in to air traffic control.

\subsection{Software for data exploration and mission support}

In our experience, in recent years available forecast data have been mainly provided by means of static or animated plots created by the scientists supporting the aircraft mission. Meteorological predictions have often been presented with the help of simple web interfaces. An early such interface is described in Flatøy et al. (2000). During recent campaigns, including POLARCAT-GRACE and CONCERT 2008 (Voigt et al., 2010), we provided access to ECMWF forecasts and Meteosat imagery through an interface allowing the user to view up to two synchronised loops of the products. Figure 2 shows the web interface with an example.

Institutions other than DLR that conduct airborne atmospheric measurements include the American National Center for Atmospheric Research (NCAR) and the National Aeronautics and Space Administration (NASA). NCAR operates so-called field catalogues ${ }^{3}$. On these web sites, forecast and observational data from satellites and radar can, similar to our web site, be viewed in loops of pre-computed images. The web pages also serve as a collection of links relevant to each campaign.

While providing predefined batch imagery through a website has the advantage of having an easy-to-use interface that can be accessed from any computer with a browser and Internet access, the approach has the disadvantage that the possibilities to interact with the data are very limited. More freedom in the exploration of the predictions, in controlling ondemand computations and, in particular, a possibility to plan the flight route in direct relation to the available data would make the support of research missions much more efficient.

NASA has developed the Real Time Mission Monitor (RTMM, Blakeslee et al., 2007), and, in collaboration with the University of Alabama, Huntsville, the Waypoint Planning Tool (WPT, He et al., 2010). The RTMM integrates realtime information from the NASA research aircraft, auxiliary observation platforms (satellites, radar), and other Earth science datasets into a GoogleEarth interface to improve the experiment's real-time situational awareness. The Java-based WPT is a recent development that is in parts similar to the tool we are presenting in this paper, allowing the user to interactively define a flight route on maps of provided atmospheric data.

The use of larger operational meteorological workstation systems, such as used by national and private weather agencies (e.g. $\mathrm{NinJo}^{4}$, or VisualWeather ${ }^{5}$ ), for data exploration, poses difficulties. Problems include the complexity and cost in adopting these systems to the requirements posed by aircraft research missions, their involved usage, and their high demands with respect to hardware resources.

An alternative is the application of less heavy exploration tools such as, for instance, Unidata's Integrated Data Viewer (IDV Murray et al., 2003, 2009) or the ECMWF Metview workstation (Russell et al., 2010; Siemen et al., 2010). However, these systems have until recently required the transfer of the prediction data to the computer they run on. They do not provide direct functionality to draft a flight track, either.

\footnotetext{
${ }^{3} \mathrm{http}: / /$ catalog.eol.ucar.edu

${ }^{4}$ http://www.ninjo-workstation.com

${ }^{5} \mathrm{http}: / / \mathrm{www}$. iblsoft.com/products/visualweather
} 


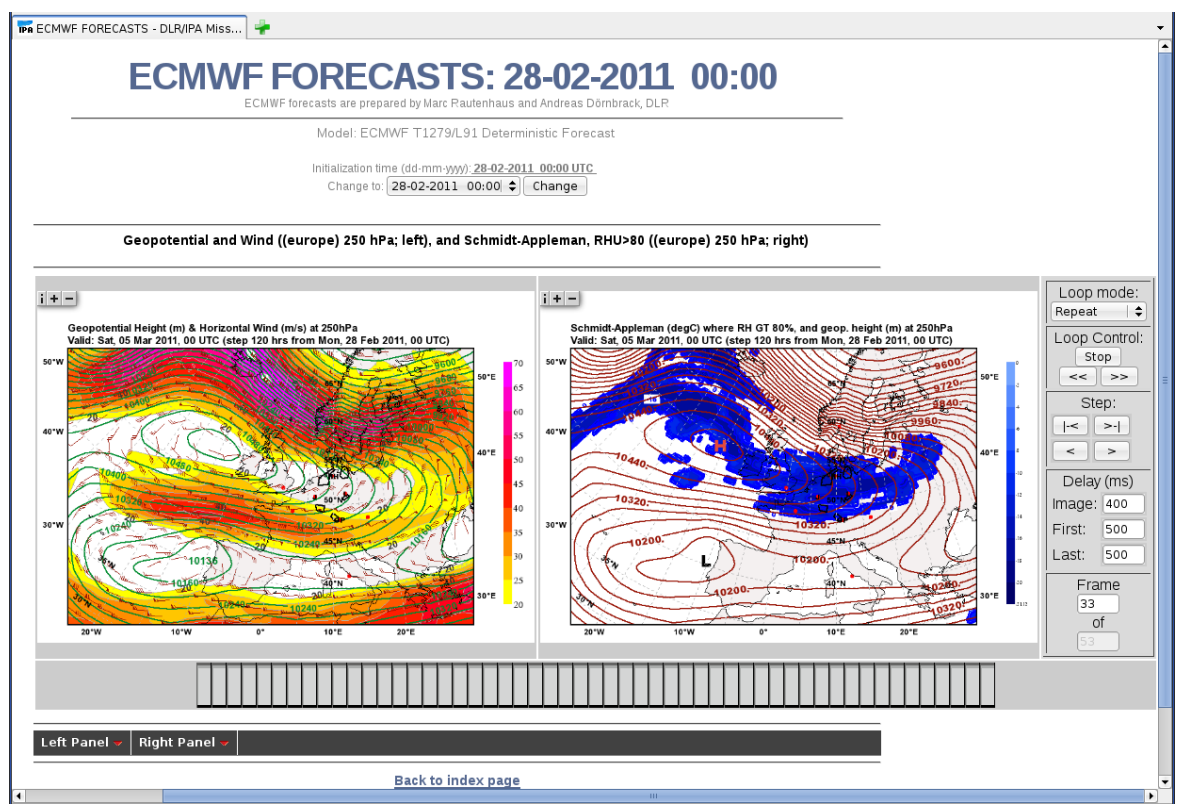

Fig. 2. DLR/IPA website providing access to batch imagery of atmospheric forecasts, satellite and radar observations. The two image loops can be synchronously time-navigated by moving the mouse over the light grey navigation bar below the images. Shown are geopotential height and wind speed on the left and the Schmidt-Appleman criterion indicating conditions for contrail formation on the right.

\subsection{OGC web services}

An interesting development closing the gap between providing static batch imagery through a web interface and rendering locally available data in a meteorological workstation is the OGC Web Map Service standard. The OGC maintains a number of widely accepted standards for geospatial data formats and web services. These include the WMS specification for accessing images of maps, the Web Feature Service (WFS) for accessing vector data, the Web Coverage Service (WCS) for accessing raster data and the Web Processing Service (WPS). A large number of both commercial and open source GIS (Geographic Information System) tools support the OGC standards to achieve interoperability for geospatial data.

The basic principle of the WMS is illustrated in Fig. 3: a client can request images of maps of geospatial data through a simple HTTP-based interface. By specifying a product layer (a dataset available from the service), parameters describing the layer (e.g. time, elevation and visualisation style) and parameters describing the map image to be returned (e.g. map projection, bounding box, image type), the client can obtain an on-demand generated image of a product. The data volume returned to the client is restricted to the image file containing the generated map. The standard in its latest versions (1.1.1 and 1.3.0) includes the mandatory operations GetMap and GetCapabilities for retrieving the maps and an XML document describing the datasets that can be visualised by the WMS. For the purpose of mission planning, the principle thus allows for the separation of user interface (client) from visualisation logic (server) as required at campaign sites with slow Internet access.

In the atmospheric sciences, OGC Web Services have gained increased attention in recent years as a way to exchange meteorological information in a standardised way (cf. the topics discussed at the three Workshops on the use of OGC Web Services in Meteorology 2008, 2009 and 2010, hosted by the ECMWF, Meteo-France and the UK Met Office, respectively). Of high interest, especially to national meteorological authorities and data vendors, are the WMS and WCS interface specifications. However, in particular for WMS, several problems have been encountered in encoding, for instance, time and space dimensions, plotting styles of the generated images, and in requesting vertical profiles and cross-sections. Strong efforts are currently under way to accommodate properties specific to meteorology in the standards. For this purpose, the Meteorology and Oceans Domain Working Group (MetOceanDWG) has been created within the OGC in 2009 (for a summary of current activities see Little, 2010; Voidrot-Martinez and Little, 2010).

Current developments for the atmospheric domain are manifold and range from open source tools to the professional workstation systems. For instance, Unidata's THREDDS data server ${ }^{6}$ features a WMS interface, ECMWF hosts activities to develop a fully web-based forecaster workstation (Raoult et al., 2010) and to support OGC Web Services in Metview (Kertész et al., 2010; Russell et al., 2010),

\footnotetext{
${ }^{6}$ http://www.unidata.ucar.edu/projects/THREDDS
} 


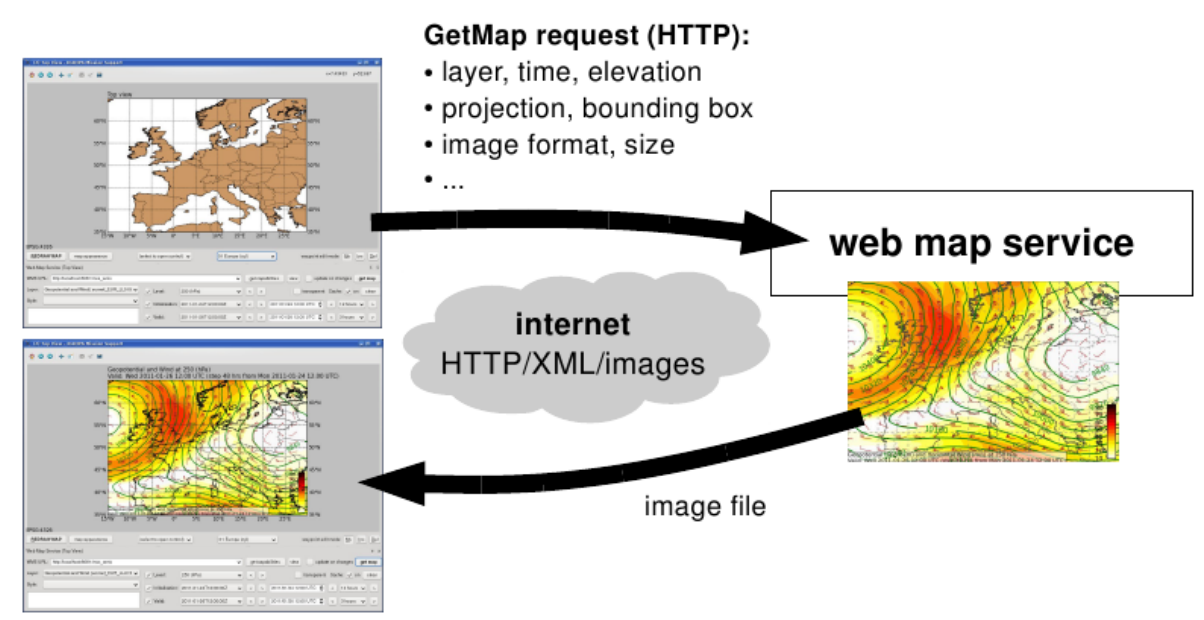

Fig. 3. Basic principle of the OGC Web Map Service standard. A client (left) sends a GetMap request, encoded as an HTTP URL, to the service (right). Within the request, the client can specify the layer and several parameters specifying the image it wants to retrieve. The server creates an image file of the requested layer and returns it to the client.

and professional workstation systems including NinJo and VisualWeather are also extended to support the OGC standards (Eymann, 2010; Matula, 2009). We refer to the homepage of the MetOceanDWG ${ }^{7}$ for further information on the status of OGC Web Services in meteorology. The page also contains links to the presentations given at the above mentioned workshops, including information on other current development activities.

\section{Architecture}

The Mission Support System is implemented in the Python programming language ${ }^{8}$ and makes use of several open source libraries including NumPy (van der Walt et al., 2011) and $\mathrm{SciPy}^{9}$ for data processing, Matplotlib with its basemap toolkit (Hunter, 2007; Tosi, 2009) for visualisation, and PyQt4 (Summerfield, 2007) for the graphical user interface (further details are given in Sect. 3.1 of the Supplement). To give the reader an impression of the look of our tool, Fig. 4 shows screenshots of the major elements of the Mission Support User Interface (MSUI), the lightweight client application with which the flight planner interacts. Forecast and flight track data is presented in a number of perspectives including horizontal and vertical views and a table listing the flight track waypoints. The user can interact with all these elements.

Figure 5 illustrates the high-level architecture of the MSS and the data flow in the system. To remain flexible throughout the development process and for future extensions, modularity was appointed a high priority in the design of the

\footnotetext{
${ }^{7}$ http://external.opengis.org/twiki_public/bin/view/ MetOceanDWG

${ }^{8}$ http://www.python.org

${ }^{9} \mathrm{http} / / / \mathrm{www}$. scipy.org
}

software. Incoming forecast and observational datasets are collected on a server (which, in our setup, is located at DLR). The server also hosts the data-processing software and the web services. The mission scientist runs the MSUI on his local computer. For interactive forecast exploration, the MSUI requests imagery from the Web Map Service, data transfer to the client is hence restricted to the image files. Thirdparty WMS sources can also be accessed by the MSUI, as well as third-party clients be used with our WMS. To investigate how flight performance computations can be integrated into the system, we have designed a prototype of a web service that computes a performance estimate for the HALO aircraft. Furthermore, although the WMS implementation provides on-demand plotting capabilities, we continue to use batch generated imagery in order to have loops of important products ready for fast exploration as soon as a new forecast becomes available. Access to a number of such predefined forecast products, as well as satellite and radar products, is available through the web page of our system (shown in Fig. 2), as well as through an MSUI module providing extended exploration functionality. In this section, we briefly describe the individual modules of the MSS. More detailed information about implementation and usage of the system are provided as Supplement to this article.

\subsection{Web Map Service}

As mentioned in Sect. 2.3, some issues have to be considered when using the WMS standard for atmospheric data. In its recent versions 1.1.1 and 1.3.0, WMS only supports one time dimension. However, atmospheric predictions usually feature two time dimensions, the forecast run initialisation time (or base time) and the time at which a forecasted field is valid. Although the WMS standard allows to define custom dimensions, adding a dimension for the initialisation time 


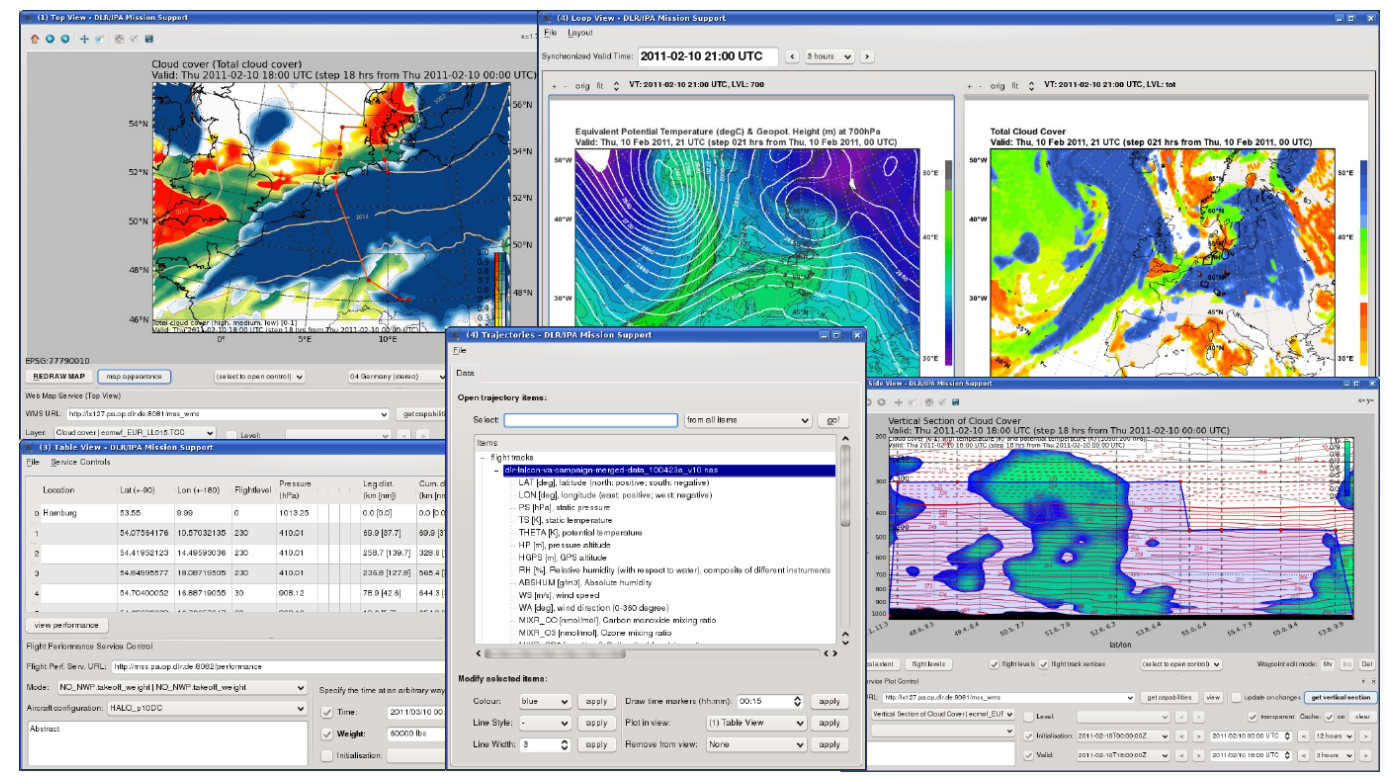

Fig. 4. Modules of the Mission Support User Interface (MSUI) application: top view (upper left), loop view (upper right), table view (lower left), trajectory tool (lower middle) and side view (lower right).

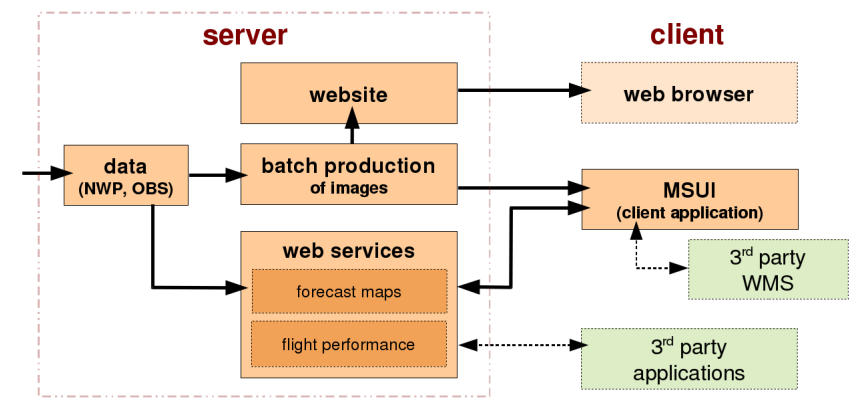

Fig. 5. The high level architecture of the tool. Incoming data (numerical weather predictions and observations) are visualised by batch jobs (predefined maps) and a web map service (WMS, ondemand). Batch products can be viewed by means of a web page and the Mission Support User Interface (MSUI) application. The MSUI can also access the WMS and the prototype of a flight performance service. The dashed lines indicate where interfaces to third-party resources exist (WMS of horizontal maps).

raises interoperability issues. First, to remain interoperable, all services offering atmospheric prediction data need to use the same identifier for the initialisation time dimension. Second, if the valid times of all initialisation times are combined in the time dimension, not all combinations of initialisation and valid time may be available. The MetOceanDWG currently discusses an approach in which the valid time is defined as a time difference with respect to the initialisation time (the lead time, for instance, if the initialisation time was 2011-03-01, 00:00 UTC, the forecast valid time 201103-02, 12:00 UTC would be expressed as “36h"), requiring, however, that the client can interpret the initialisation time dimension. Until this problem is resolved in the standard, our system uses the following simple approach: we added the dimension INIT_TIME. Both this new dimension and the time dimension are given as true time (as strings that take the format 2011-03-01 T00:00:00 z). When a client requests an invalid combination, a service exception is returned.

Figure 6 shows an excerpt from a capabilities document generated by our service (corresponding to version 1.1.1 of the WMS standard), including both time dimensions. For the displayed layer, a vertical pressure coordinate in units of $\mathrm{hPa}$ is defined in the ELEVATION dimension. For other data types, this dimension can also specify model levels or be omitted. The MSS WMS currently provides maps in a standard equidistant cylindrical projection (encoded with the WMS typical EPSG: 4326 code). Further projections required for specific campaign setups can be defined as long as they are supported by Matplotlib's basemap toolkit. Note that the handling of parametrised projections (including stereographic projections) is also a topic under discussion in the MetOceanDWG.

To provide vertical cross-sections along a flight track path, we extended the service by a few non-standard parameters. An approach we found usable was to request a cross-section with a vertical logarithmic pressure axis by specifying a coordinate reference system VERT : LOGP, then using the BBOX argument to define the vertical extent of the section and the number of interpolation points to be used. A new parameter PATH was introduced to pass the waypoints of the path of the vertical section. The "bounding box" (which actually is not a bounding box anymore) is given in the format $\mathrm{BBOX}=100,1025,0,200$ to request, in this example, 


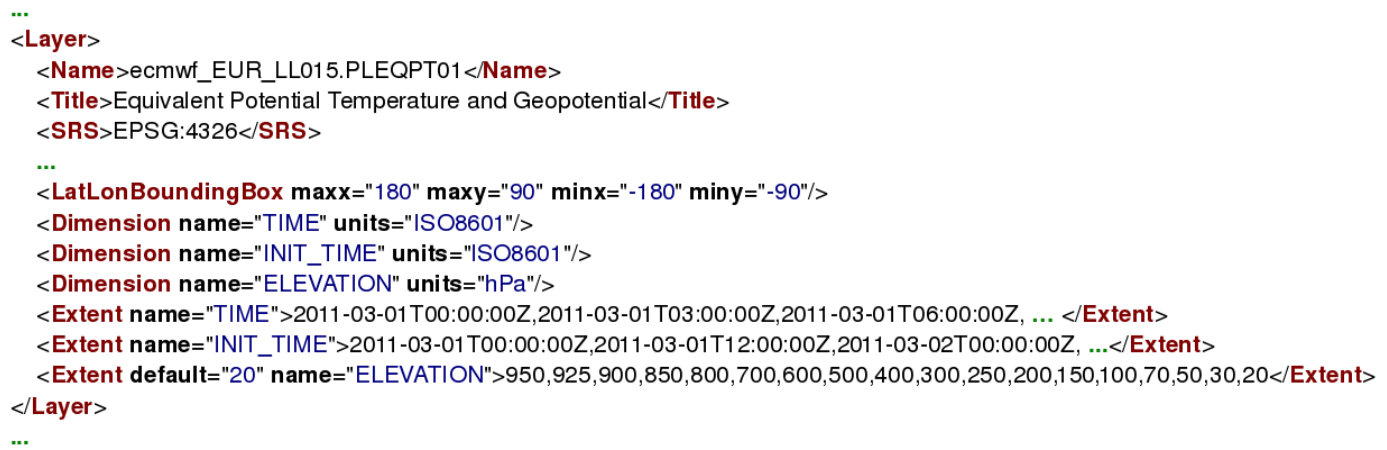

Fig. 6. Excerpt from a capabilities document returned by the Web Map Service (WMS version 1.1.1) for a sample layer visualising equivalent potential temperature and geopotential height.

http://mss.pa.op.dlr.de/mss_wms

\&layers=ecmwf EUR LL015.TCC\&styles=default

\&srs=EPSG:4326\&format=image/png

\&height $=400$ \& width $=600$ \&bbox $=-15.0,35.0,30.0,65.0$

\&dim init time=2011-03-02T00:00:00Z\&time $=2011-03-03 T 12: 00: 00 Z$

\&exceptions=application/vnd.ogc.se_xml\&transparent=FALSE

http.//mss.pa.op.dlr.de/mss wms

?request $=$ GetMap\&version $=1.1 .1$

\&layers=ecmwf EUR LL015.TCC\&styles=default

\&srs=VERT:LOGP\&format=image/png

\&height $=280 \&$ width $=600$ \&bbox $=101,1050.0,10,175.0$
\&path $=48.08,11.28,41.30,11.28,41.30,9.18,48.78,9.18$

\&dim init time $=2011-03-02 \mathrm{T00}: 00: 00 \mathrm{Z} \&$ time $=2011-03-03 \mathrm{~T} 12: 00: 00 \mathrm{Z}$

\&exceptions=application/vnd.ogc.se_xml\&transparent=FALSE
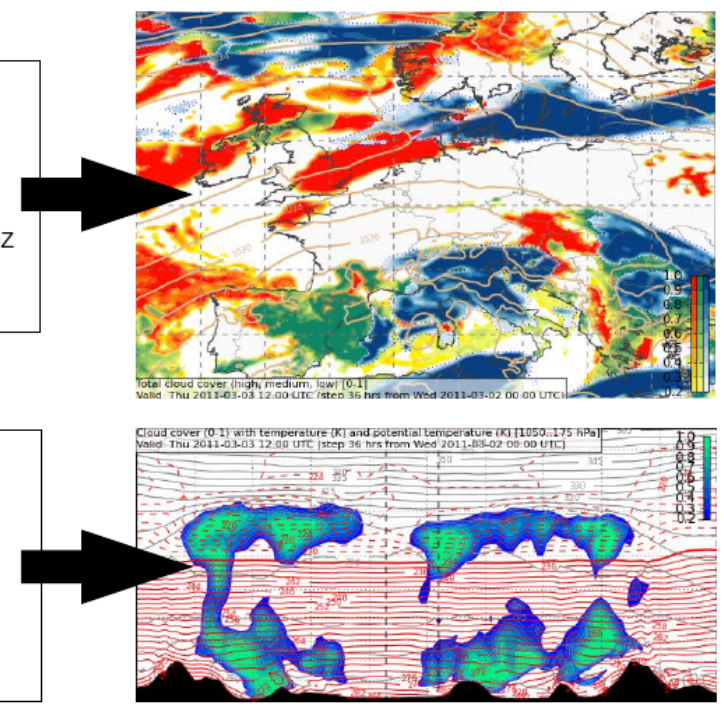

Fig. 7. Examples of two WMS requests. At the top, a product of predicted cloud cover is requested (high clouds in blue, medium level clouds in green and low clouds in red). Note the usage of the INIT_TIME dimension to specify the forecast initialisation time. At the bottom, a vertical cross-section of the cloud cover product is requested. Note the BBOX parameter that is used to specify the vertical extent of the section (in pressure coordinates) and the number of interpolation points to be used, and the additional PATH parameter.

a cross-section with a vertical axis ranging from $1025 \mathrm{hPa}$ to $200 \mathrm{hPa}$ which is interpolated at 100 points between the two end points. $\mathrm{PATH}=40,10,50,10,50,15$ is interpreted as a cross-section path connecting the three points at $40^{\circ} \mathrm{N}, 10^{\circ} \mathrm{E}, 50^{\circ} \mathrm{N}, 10^{\circ} \mathrm{E}$ and $50^{\circ} \mathrm{N}, 15^{\circ} \mathrm{E}$ using the shortest route between the points. Hence, the 100 interpolation points are generated along great circles between the specified waypoints.

Figure 7 shows sample requests for a map and a vertical section and the resulting images. We have created a set of standard products that visualise combinations of NWP fields that have proven to be useful in the practise of mission planning. They include, for instance, geopotential height combined with wind speed and wind barbs or with the equivalent potential temperature, or high, medium and low level cloud cover overlaid with mean sea level pressure. The service is capable of generating plots of individual fields as well. However, from our experience we find that combining fields in one product often makes the visualisation clearer. The alternative, requesting multiple images and overlaying them in the client, is more flexible, but may lead to features in one image being obscured by another (e.g. contour labels). Note that our styles include a small text field (showing the name of the product, initialisation and valid time) and the legend within the returned map or cross-section.

We note that our goal for using the WMS standard was not to create a service and client that can handle all aspects of the standard. Instead, we regard its usage as a beneficial approach to providing on-demand visualisations of large forecast datasets that cannot be transferred to the campaign site in 


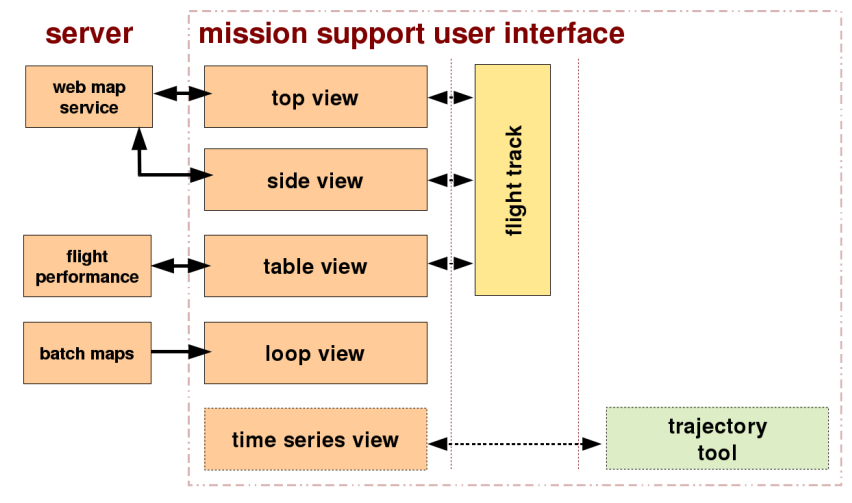

Fig. 8. The architecture of the Mission Support User Interface application.

total (for instance, an ECMWF forecast dataset as described in Sect. 4, covering a horizontal area of $40^{\circ} \times 100^{\circ}$ on a regular latitude/longitude grid with a resolution of $0.15^{\circ}$, encompasses about 20 gigabytes if stored in NetCDF format ${ }^{10}$ ). The approach also yields the advantage of interoperability, for example, being able to access third-party WMS sources useful for flight planning. However, issues we specifically did not address in the present work include the dynamic update of capabilities and imagery, the GetFeature Info request, providing legends as separate images, multiple layer requests, and providing other plot types such as vertical profiles or time series. Nevertheless, our software is ready to be adopted to new standard elements (as worked on in the MetOceanDWG), so it will be straightforward to extend the interoperability with other providers offering atmospheric data via WMS.

Regarding the computational performance of the WMS implementation, the creation of a horizontal or vertical crosssection image takes between three and eight seconds on a single core of an Intel Core- 2 running at $2.6 \mathrm{GHz}$. To minimise the image file sizes, the produced images are converted to indexed colour with an 8-bit colour palette. An $800 \times 600$ pixel image, a typical image size requested by the MSUI, consequently takes on the order of 150-200 kilobytes if transmitted as a compressed PNG (Portable Network Graphics) file. In comparison, the raw data fields for the plot shown in Fig. 9 (the three variables geopotential height and $u$ and $v$ wind components, stored as 32-bit floating point numbers on a regular latitude/longitude grid covering a horizontal area of about $30^{\circ} \times 50^{\circ}$ with a resolution of $0.15^{\circ}$ ) encompass about 800 kilobytes. Hence, in terms of data volume, extracting the raw data fields for a plot on the server and transmitting these in compressed form for client-side visualisation would be of the same order of magnitude. However, this approach would be less flexible in terms of interoperability and require more complicated client logic for the visualisation. The latter

\footnotetext{
${ }^{10} \mathrm{http}: / /$ www.unidata.ucar.edu/software/netcdf
}

would be disadvantageous when, for instance, to include data of a new type, both server and client software would need to be modified.

\subsection{Flight performance web service}

Two questions often arise in flight planning: is the flight track technically feasible in terms of total distance and vertical profile? How long is it going to take to arrive at a certain waypoint? To address these questions, we have implemented a prototype of a web service computing an estimate of the flight performance of the German HALO aircraft.

Several parameters can influence the performance of the aircraft. The atmospheric conditions, in particular wind but also temperature influence the time and fuel required for a given segment. The weight of the aircraft (as determined by the payload and the loaded fuel) affects fuel consumption and the flight level that can be reached (high flight levels cannot be reached with a heavy aircraft).

The flight performance is computed with a lookup-table approach. The lookup-table lists possible cruise speeds and fuel consumption for a given aircraft weight, flight level and atmospheric conditions. To determine the feasibility of a flight route, the route is split into small segments for which the required time and fuel are computed. This way, it is possible to make statements on whether the planned flight route is technically feasible and to estimate the approximate time and fuel consumption.

Note that for safety and legal reasons, the flight performance computation cannot replace the computations the pilots have to carry out before takeoff. However, it can serve the scientist planning the flight as an indication for whether the flight is feasible and how long it will approximately take.

The performance module is implemented, similar to our Web Map Service, as a WSGI application. Since, to the best of our knowledge, no similar services exist that would be advantageous to be interoperable with, we designed an interface very close to the WMS specifications. In particular, we use a modified GetCapabilities to communicate the allowed parameter ranges the service offers, and a GetPerformance request similar to the WMS GetMap request to obtain the performance results. This approach has the advantage that we are able to re-use methods from the WMS implementation for the Flight Performance Service, for instance, to create and parse the capabilities document. The flight track is passed to the service with an HTTP request similar to the vertical cross-section WMS request. Results of the performance computations are returned to the client by means of a text file. Further details on the service can be found in the diploma thesis by Bauer (2011).

\subsection{Client application}

The architecture of the MSUI is illustrated in Fig. 8. The central elements are the views, application modules that allow 


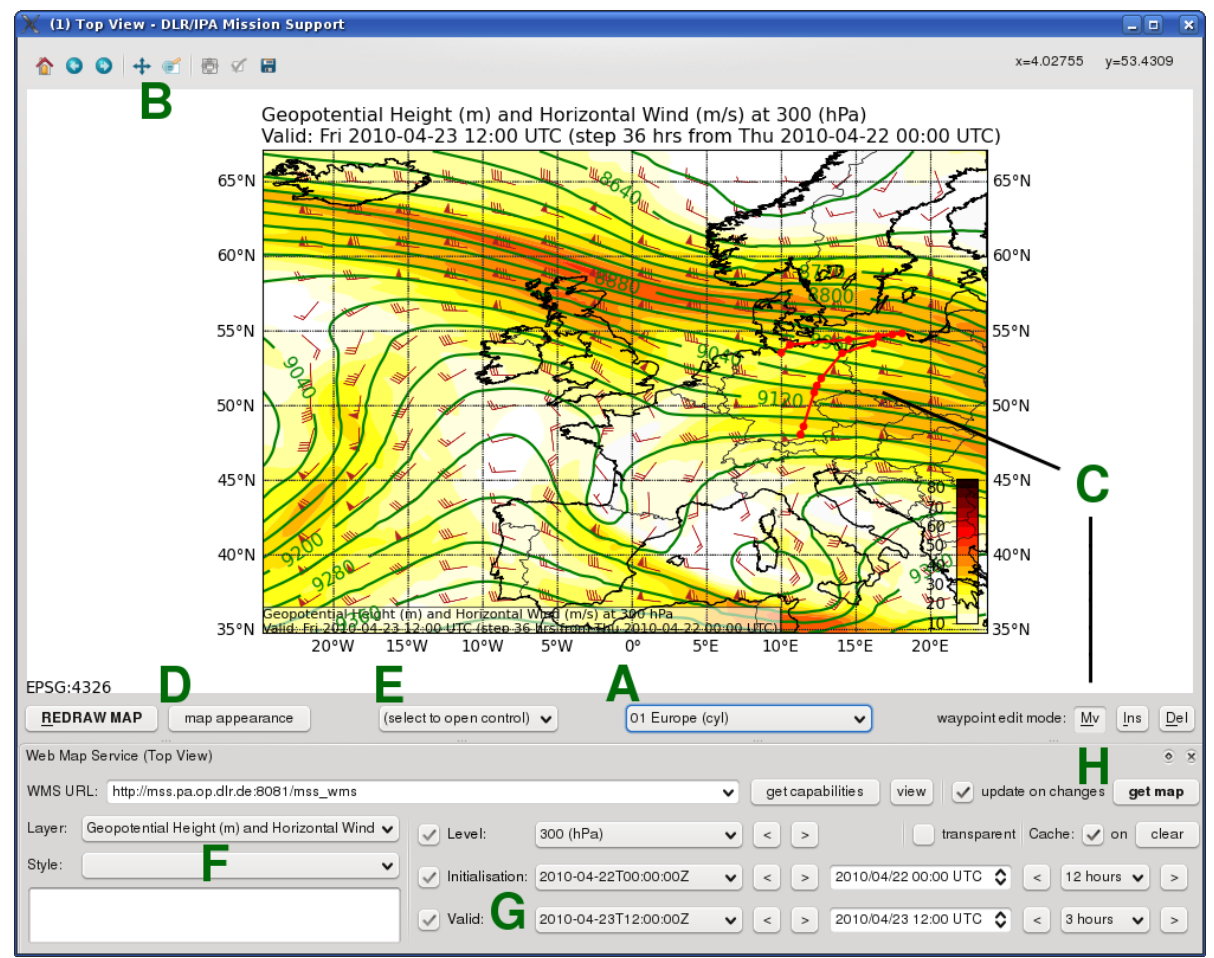

Fig. 9. Screenshot of the Mission Support User Interface Top View. The labels mark (A) selection of map projection and section, (B) zoom/pan, (C) interaction with the waypoints of the flight track, (D) customisation of map appearance, (E) open controls, (F) selection of WMS layer and style, (G) WMS time navigation, (H) trigger a WMS GetMap request. Shown is a map of ECMWF-forecasted geopotential height $(\mathrm{m})$ and horizontal wind speed $\left(\mathrm{m} \mathrm{s}^{-1}\right)$ at $300 \mathrm{hPa}$ on 23 April 2010, 12:00 UTC. Overlaid is the flight track discussed in Sect. 4.

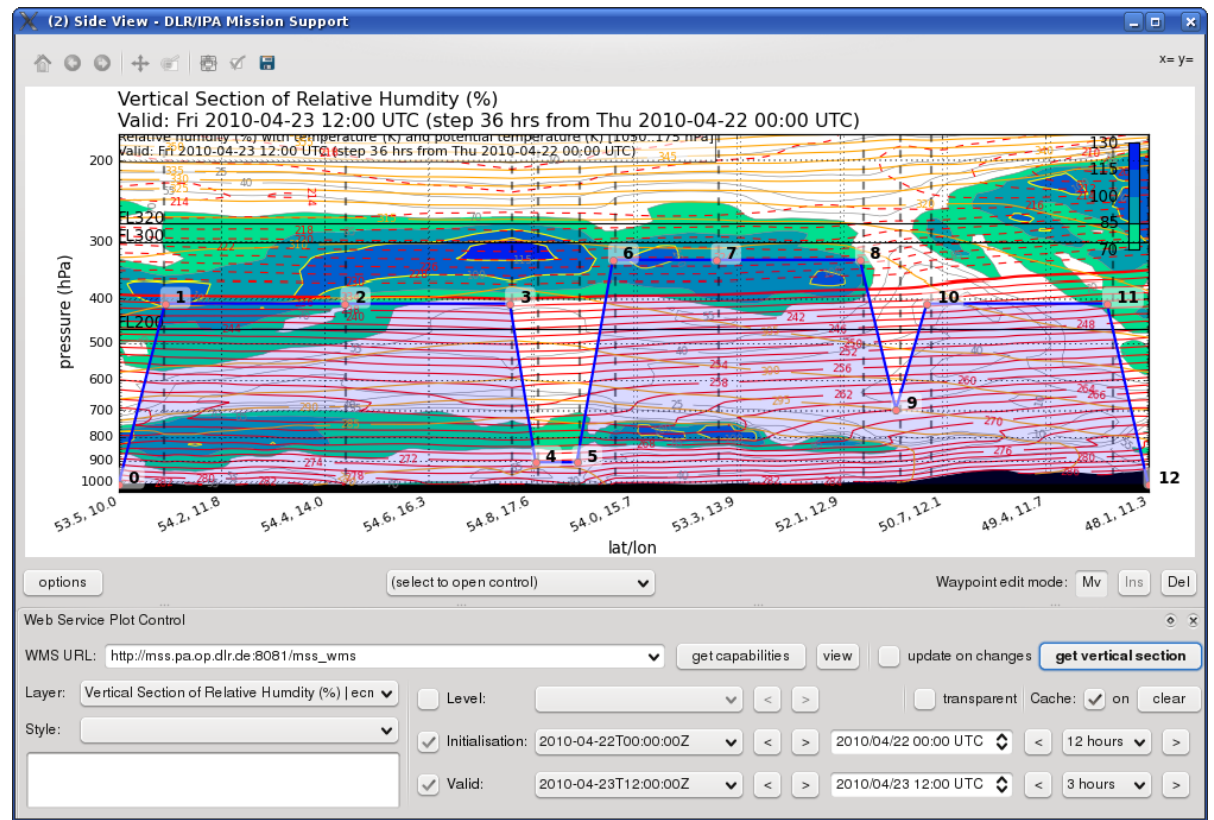

Fig. 10. Screenshot of the Mission Support User Interface Side View. The same WMS client control as in the top view is used to request the vertical cross-section images. Shown is a cross-section of ECMWF-forecasted relative humidity on 23 April 2010, 12:00 UTC along the flight track discussed in Sect. 4. 
the user to view a data model, in particular the flight track, from different perspectives. Three views allow interacting with the flight track. A top and a side view provide a view on the horizontal flight route and the vertical flight profile, respectively, with access to imagery obtained from a WMS. In the top view, the horizontal position of the flight track waypoints can be interactively changed, in the side view the flight level. A table view shows the coordinates of the waypoints, computes the length of the track and allows to query the flight performance web service. A fourth view, the loop view, provides access to batch imagery.

Tools in our architecture are modules that allow to manage additional data models and that do not provide visualisations. For instance, we implemented a trajectory tool that allows to load time series of past research flights and trajectory data from the LAGRANTO model. A corresponding time series view provides the visualisation functionality.

Similar to other Geographical Information Systems, the top view contains a map with which the user can interact. Figure 9 shows a screenshot of the module. A number of predefined map projections and map sections are available, in addition it is possible to zoom and pan to any arbitrary region. The waypoints of the flight track can be dragged, deleted, and new ones inserted. In consistency with our WMS extension, they are connected by great circles. Controls are modules that allow data from external sources to be loaded into a view. Two controls can be opened to load additional data into the top view, a WMS client and a module to load predicted satellite tracks obtained from the web-based NASA LaRC Satellite Overpass Predictor ${ }^{11}$. The WMS client provides twofold controls for time navigation. First, the time values extracted from the capabilities document are presented to the user (the left-hand elements displaying time information in Fig. 9). This approach is intended to work with any Web Map Service, even if the times provided by the service are given in a format unknown to the client. Second, the client tries to interpret these time values to allow for more convenient time navigation (the right-hand elements displaying time information). The benefit is that the user, instead of only navigating to the next or previous time step, can choose an arbitrary time interval (for instance, $24 \mathrm{~h}$ ). Also, the valid time is kept fixed when the initialisation time is changed and vice versa. This way, it is possible to switch back and forth between different forecast model initialisations to explore, for example, whether the movement of a front has changed between two forecasts. Retrieved images are stored in a file cache in order to accelerate the retrieval of already requested maps.

The side view (Fig. 10) allows to specify the vertical profile of the flight track and to view vertical cross-sections. It is designed in a similar fashion to the top view, with the WMS client control used to retrieve cross-section imagery. The vertical axis of the side view is given in pressure coordinates,

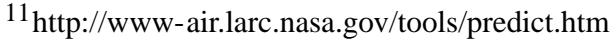

axis limits and view appearance can be customised. For orientation, the location of arbitrary flight levels can be superimposed on the pressure axis. Exact coordinates and flight levels of the waypoints can be specified in the table view (Fig. 11). In this module, the flight planner can obtain information on the length of the flight track and its individual segments. Furthermore, access to the flight performance web service is provided.

\subsection{Interoperability}

With regard to horizontal maps, the MSS can be coupled to third-party data providers or clients by means of the WMS interface. This is illustrated by the green boxes and dashed lines in Fig. 5. We have successfully tested the MSUI top view with several external WMS sources running, for instance, MapServer ${ }^{12}$ or GeoServer ${ }^{13}$ as mapping software (Fig. 14 gives an example in the top right panel). Hence, if a partner involved in a campaign can provide his data through a Web Map Service, the data can be readily included. Furthermore, our WMS can be accessed from third-party clients, for instance, Metview 4. However, since our WMS requires both the TIME and INIT_TIME dimensions to be defined, the client needs to allow the specification of additional dimensions.

\section{Case study}

We demonstrate the application of our tool by discussing a case study. Consider the scenario of the 2010 Eyjafjalla volcano eruption that led to a number of airspace closures in Europe. Between 19 April and 18 May 2010, the DLR Falcon aircraft performed a series of 17 flights between southern Germany and Iceland (Schumann et al., 2011). The objective was to take measurements of lidar aerosol backscatter, in-situ aerosol concentrations, trace gas mixing ratios, single particle properties, and meteorological parameters in the volcanic ash plumes. Here, we revisit the flight of 23 April 2010. On 22 April 2010, a flight had been performed along the southern Norwegian coast, after which the Falcon landed in Hamburg. For 23 April 2010, the objective was to acquire lidar and in-situ observations of the horizontal location and vertical extent of the ash cloud. For operation of the lidar, it was a requisite to design a flight track along which volcanic ash was forecasted but no water clouds. Takeoff needed to be in Hamburg and landing in Oberpfaffenhofen. Due to a required engine maintenance in the morning, takeoff was not possible before early afternoon local time.

To study how this situation could have been handled with the Mission Support System, we assume the availability of two types of prediction data, weather predictions

\footnotetext{
${ }^{12} \mathrm{http}: / /$ mapserver.org

${ }^{13} \mathrm{http}: / /$ geoserver.org
} 


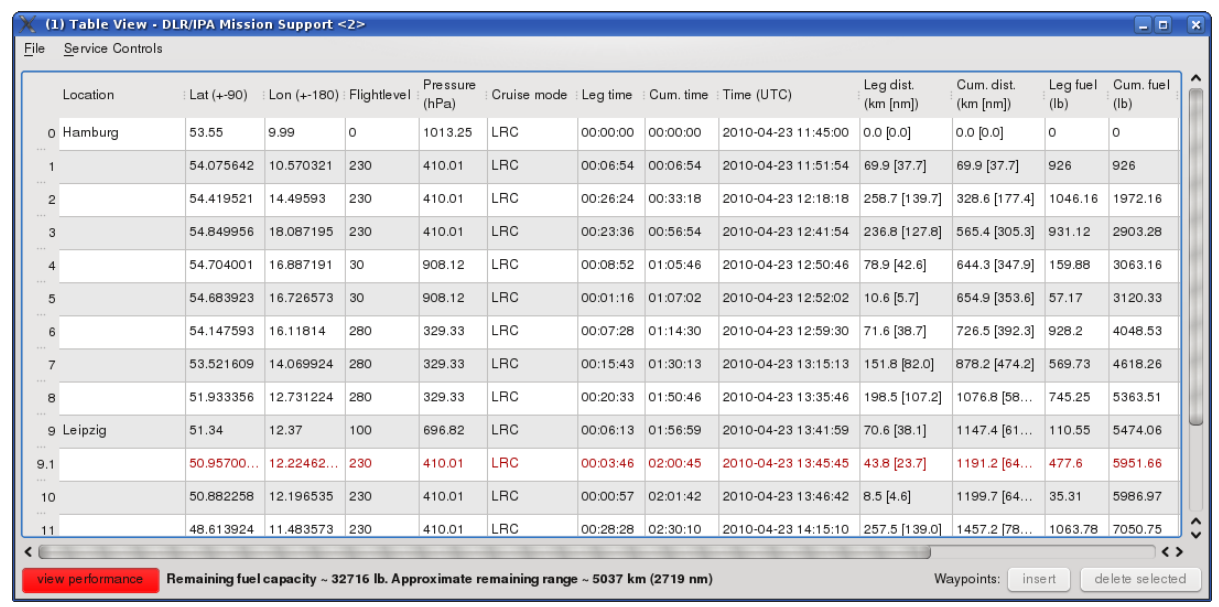

Fig. 11. Screenshot of the Mission Support User Interface Table View, listing the waypoints of the flight track discussed in Sect. 4. The table also lists flight performance values obtained from the prototype web service described in Sect. 3.2.

from ECMWF and volcanic ash cloud predictions from the ECHAM/MESSy Atmospheric Chemistry (EMAC) model.

The ECMWF predictions are obtained from the operational deterministic forecast run in T1279L91 resolution, i.e. with a spherical truncation of T1279 (corresponding to a Gaussian grid of approx. 0.15 by 0.15 degrees in latitude and longitude) with 91 vertical hybrid pressure levels up to $0.01 \mathrm{hPa}$. These data are available twice daily (00:00 and 12:00 UTC analyses) at a time step of three hours up to $144 \mathrm{~h}$ forecast lead time and six hours up to $240 \mathrm{~h}$ forecast lead time.

The EMAC model is a numerical chemistry and climate simulation system that includes sub-models describing tropospheric and middle atmosphere processes and their interaction with oceans, land and human influences (Jöckel et al., 2010). It uses the Modular Earth Submodel System (MESSy) to link multi-institutional computer codes. The core atmospheric model is the 5th generation European Centre Hamburg general circulation model (ECHAM5, Roeckner et al., 2006). For our use case EMAC was operated in the T106L31-resolution (approx. 1.125 by 1.125 degrees in latitude and longitude, with 31 vertical hybrid pressure levels up to $10 \mathrm{hPa}$ ) and nudged to ECMWF analysis data. A passive tracer was released at the Eyjafjalla location, following the source characteristics listed in Fig. 2 of Schumann et al. (2011). Although this simulation represents a reanalysis of the eruption incident, the data modalities for a true forecast simulation with EMAC would be the same.

We assume the planning activities to occur on the afternoon of 22 April 2010, i.e. the ECMWF forecast initialised at 00:00 UTC on that day is available to the flight planners. First, we use the MSUI top view to analyse the location of the ash cloud and the synoptic situation, focusing on the wind field and water cloud occurrence.
Examination of a product visualising geopotential height and wind speed on pressure levels and of a mean sea level pressure product reveals that during 22 and 23 April 2010, central Europe is influenced by weak high pressure reaching from the British Isles to eastern Europe. Surface winds over Germany are generally weak, with no preferred wind direction. South of a small low pressure system off the west coast of Norway, a west-northwesterly flow extends over Denmark and the Baltic Sea. At higher altitudes, this flow intensifies to become the jet stream, with a maximum over Scotland. The product loaded in the top view in Fig. 9 shows the $300 \mathrm{hPa}$ geopotential height and wind speed, valid on 23 April 2010, 12:00 UTC.

Next, we explore the EMAC volcanic ash product. The left panel of Fig. 12 shows the total column density of the passive ash tracer at 09:00, 12:00 and 15:00 UTC on 23 April 2010. The images are directly saved from the top view. As expected from the wind forecasts, the ash cloud observed south of Norway during the 22 April flight is advected by the westerly flow towards the Baltic Sea. Only little or no ash is predicted west and south of Hamburg. Hence, the flight needs to be planned into an easterly direction.

However, the ECMWF cloud cover prediction shows that the westerly flow also advects cloud fields towards northern Germany and the Baltic Sea. The right panel in Fig. 12 presents the forecasted cloud cover on 23 April 2010. The maps show the grid box area fraction covered by clouds in the model, divided into three vertical segments. High level clouds are plotted in blue, medium level clouds in green and low level clouds in orange. While at 09:00 UTC large areas along the German and Polish coast are cloud free, cloud cover increases during the course of the day. However, cloud cover fraction along the coast line is low, indicating broken clouds that allow for at least partial lidar measurements. 

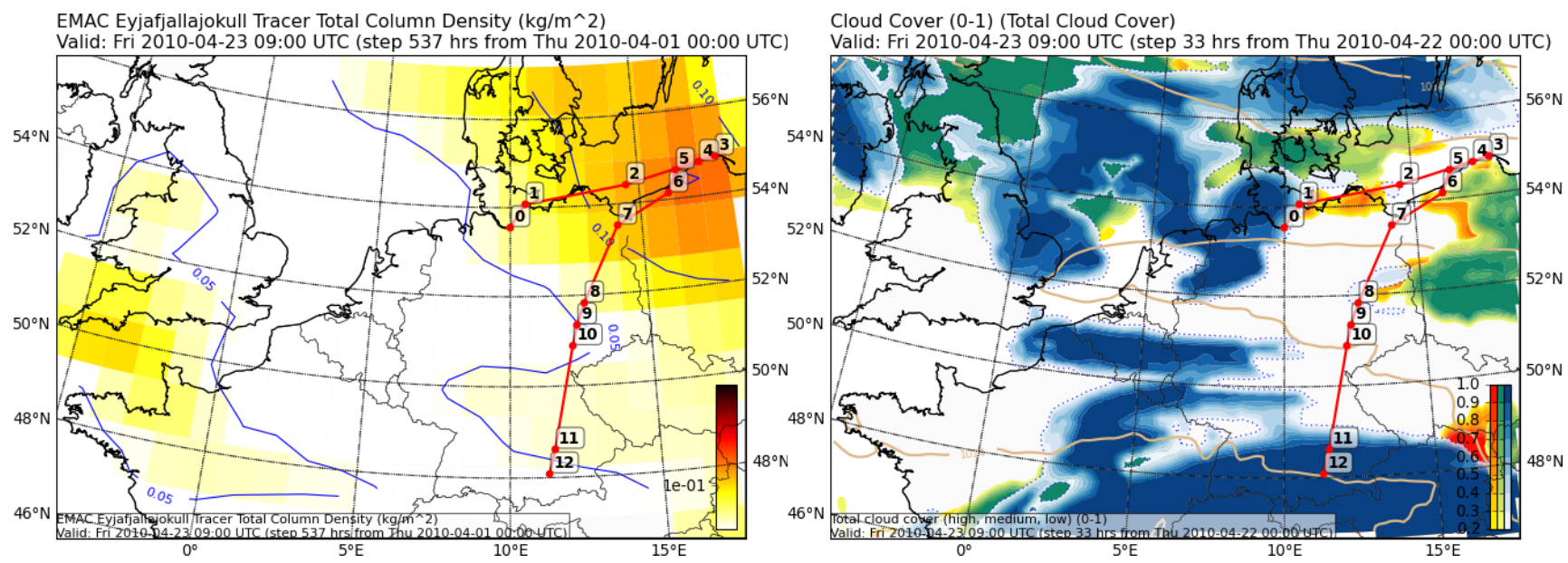

EMAC Eyjafjallajokull Tracer Total Column Density $(\mathrm{kg} / \mathrm{m} \wedge 2)$
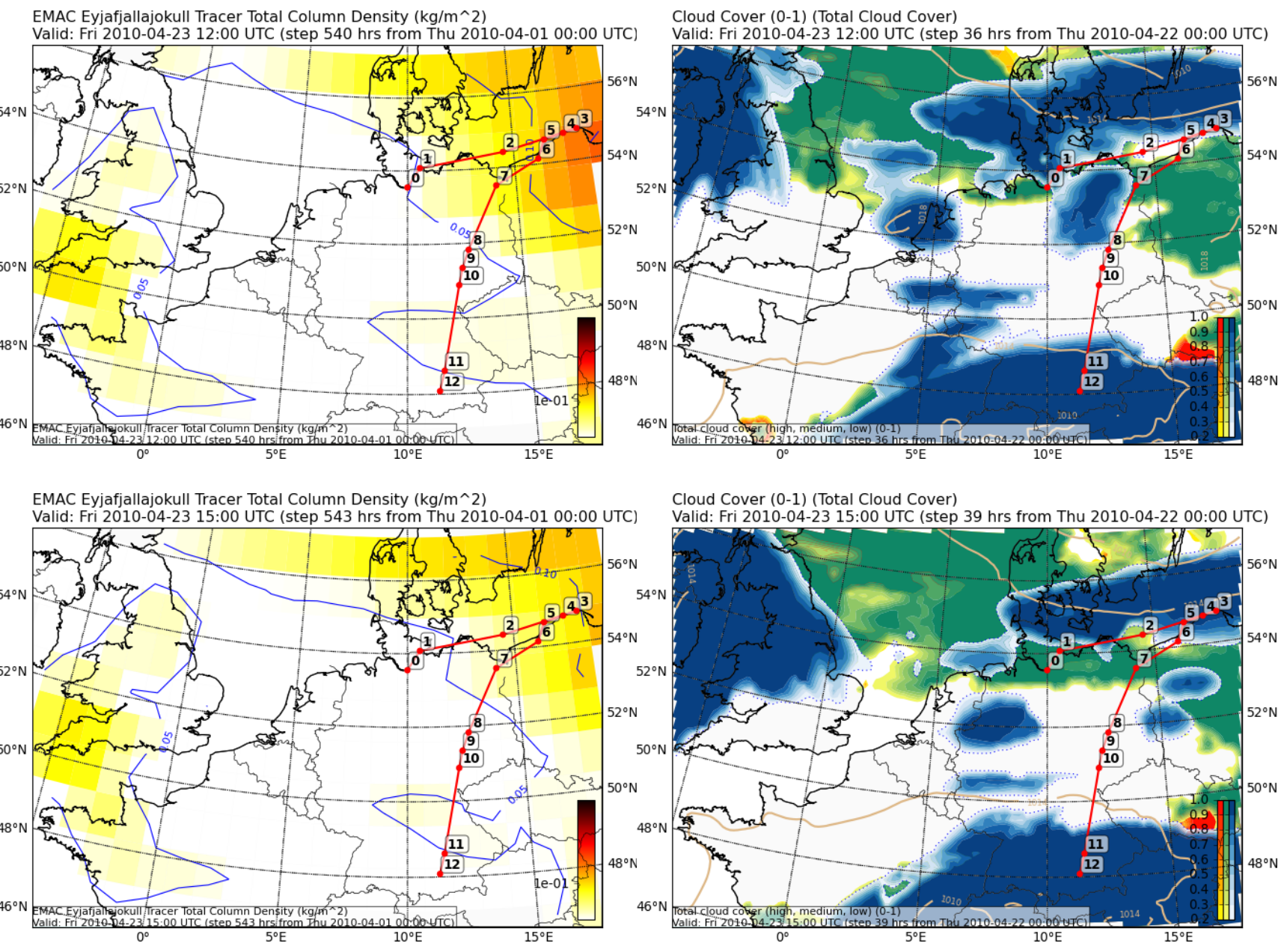

Fig. 12. Products from the MSUI top view: forecasted volcanic ash cloud (left panel) and water clouds (right panel) on 23 April 2010 at 09:00, 12:00 and 15:00 UTC. The flight track as planned in the MSUI is plotted in red. The volcanic ash cloud is visualised by the total column density $\left(\mathrm{kg} \mathrm{m}^{-2}\right)$ obtained from the EMAC output. Water clouds are shown separated into three vertical layers, blue for high clouds, green for medium level clouds, and red for low clouds. For each layer, the forecasted grid box fraction (in the range $0 . .1$ ) is plotted. 

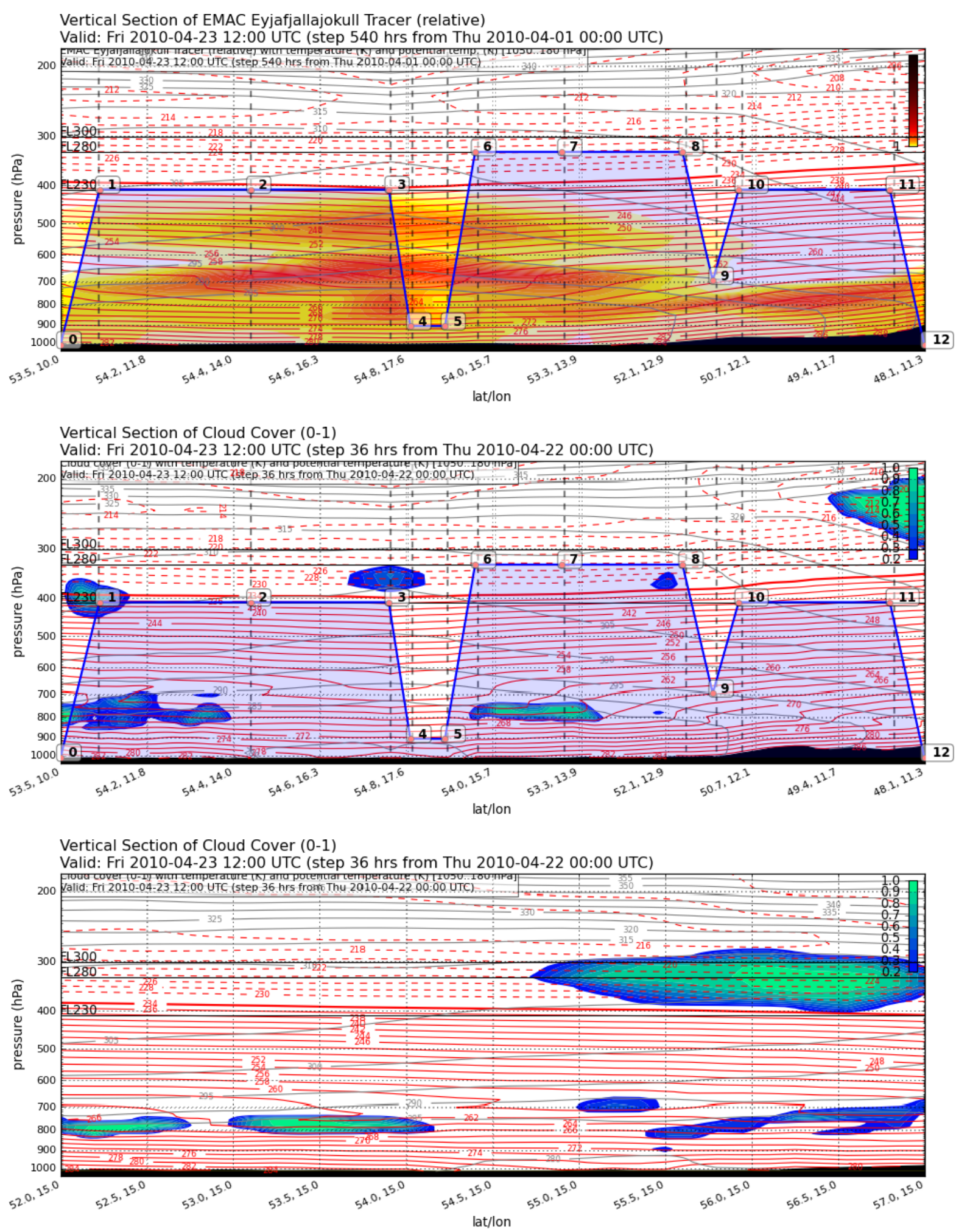

Fig. 13. Products from the MSUI side view: vertical cross-sections of forecasted volcanic ash cloud and water clouds on 23 April 2010 at 12:00 UTC. The upper two sections show the model data interpolated to the planned flight track. The bottom section shows the vertical structure of the water clouds between $52^{\circ} \mathrm{N}$ and $57^{\circ} \mathrm{N}$ along the $15^{\circ} \mathrm{E}$ meridian. 

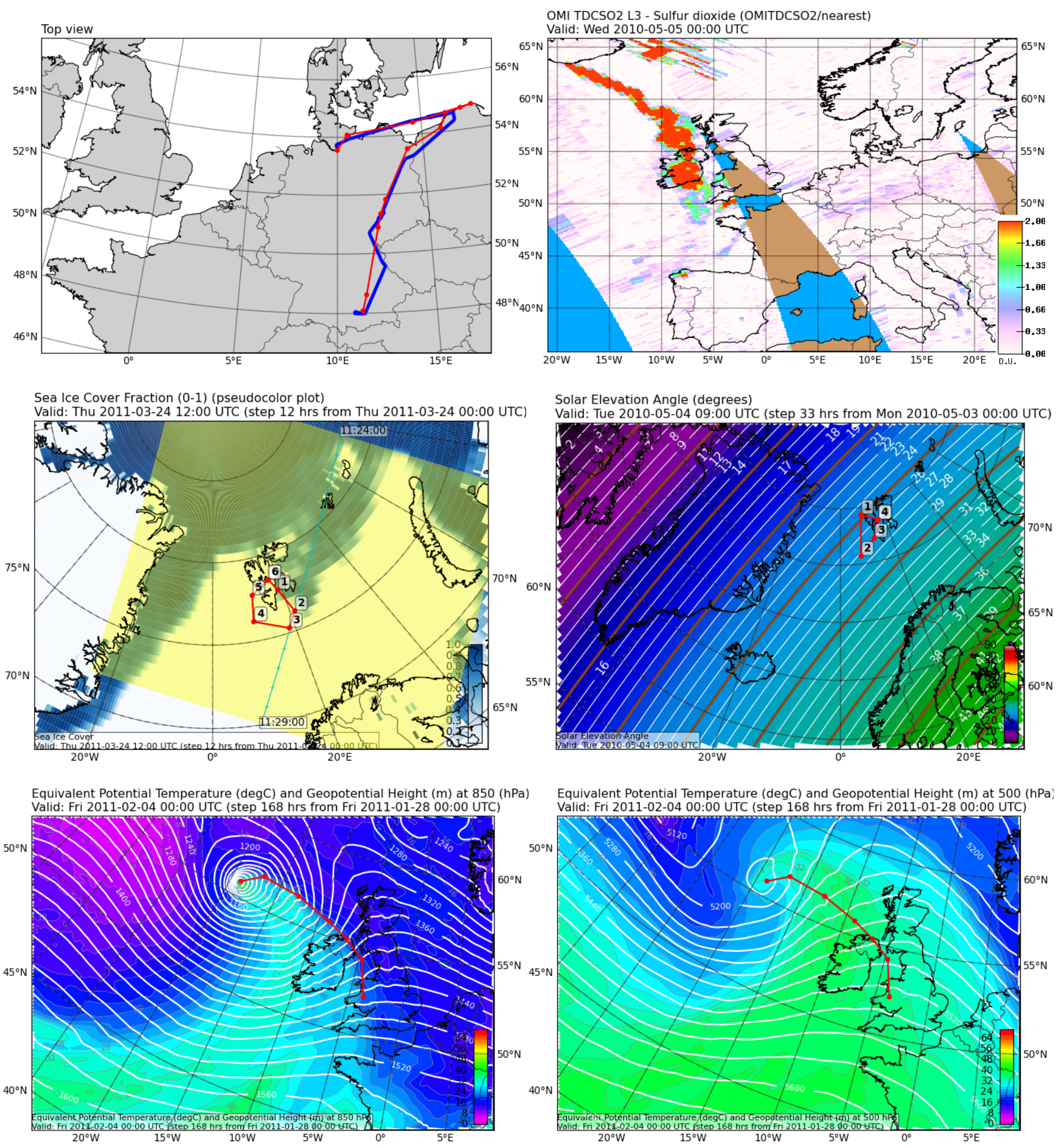

Fig. 14. Examples of further applications of the MSUI. Top left: comparison of the actually flown flight track of 23 April 2010 (blue) and the planned track (red). Top right: access to third-party WMS sources. Shown is the daily total sulphur dioxide column as measured by the Ozone Monitoring Instrument (OMI) on 5 May 2010. Note the plume emanating from the Eyjafjalla volcano. The WMS image has been obtained from a service provided by the ADAGUC project at KNMI (http://adaguc.knmi.nl), based on NASA data from OMI-AURA. Middle left: plan of a flight track in the Spitsbergen area in synchronisation with a satellite overpass (cyan line and yellow patch), while not crossing the sea ice boundary (blue grid boxes). Middle right: plot of the solar elevation angle to ensure proper function of radiation instruments. Bottom: usage of a drawn track to mark the position of a front at a given altitude. Shown are plots of equivalent potential temperature at $850 \mathrm{hPa}$ (left) and $500 \mathrm{hPa}$ (right) of a low pressure system west of Scotland. 
We start to plan a flight track running eastward along the German and Polish coast, then returning along the Polish coast and leading southward back to Oberpfaffenhofen over eastern Germany (the waypoints drawn in Fig. 12). On the way back, we intend to pass a ground-based lidar station in Leipzig. The next step is to estimate the vertical structure of ash and water clouds from vertical cross-sections. The upper two plots in Fig. 13 show the cross-sections as displayed in the side view for 12:00 UTC (note that the units of the horizontal and vertical ash products are different and hence cannot be compared). The ash cloud exhibits a maximum around $700 \mathrm{hPa}$, with a second maximum around $500 \mathrm{hPa}$ in the eastern parts of the flight track. Water cloud occurrence is low, with broken clouds around $800 \mathrm{hPa}$ in the first part of the flight track and high clouds located between 350 and $400 \mathrm{hPa}$.

To get additional information on the cloud field being advected from the northwest, we request an additional vertical section along the $15^{\circ} \mathrm{E}$ meridian from $52^{\circ} \mathrm{N}$ to $57^{\circ} \mathrm{N}$ (lower panel of Fig. 13). The cross-section shows that the clouds further north consist of high level clouds above $400 \mathrm{hPa}$ and broken low level clouds. Measurements should hence be possible as long as the aircraft stays below the high clouds.

We thus specify the vertical profile of the flight. Flight level 230 , located at approximately $400 \mathrm{hPa}$ underneath the expected high clouds and above the ash cloud, is chosen as a first flight elevation for lidar measurements. Within the return segment along the Polish coast (waypoints 3-6), we plan for a vertical profile and in-situ measurements. On the way back, the aircraft shall climb back to a higher flight level, with another vertical profile above Leipzig.

The table view lists a flight track length of $1520 \mathrm{~km}$. As described in Sect. 3.2, the prototype of the flight performance web service only supports the HALO aircraft. We perform a test query to learn about the feasibility of the flight track with this carrier. When carried out with HALO, the flight is estimated to take about 2:50 h. Performance values of the first part of the flight are listed in Fig. 11.

Since the Mission Support System does not account for legal constraints such as possible airways the aircraft can take, the final flight track planned by the pilots will always differ to some extent from the flight track planned in the MSS. However, our system represents a first step towards simplifying the process of drafting a flight track under consideration of the available forecasts. The resulting plots and waypoint lists can easily be communicated to the aircraft crew.

Figure 14 shows a comparison of the flight track planned in this example and the actual flight track of the 23 April 2010 flight (the actual Falcon flight took 3:10 h), as well as examples of further applications of the system. For instance, the flight track line in the top view can also be used to mark the position of an atmospheric feature such as a front. Such labelling can help to explore the vertical structure of the feature or its movement in time. As an illustration, the plots in Fig. 14 expose the tilted nature of the front, at $500 \mathrm{hPa}$ the frontal line is ahead of its position at $850 \mathrm{hPa}$.

\section{Conclusions and outlook}

We have developed a tool, the Mission Support System, to plan atmospheric research flights. The MSS allows to interactively design of a flight route in direct relation to atmospheric prediction data and, in a prototypical version, estimate the expected flight performance. It is based on web services and implemented in the open-source programming language Python. With our development, we are now able to design flight routes based on data from numerical atmospheric models in a way previously not possible. We thereby fill a crucial gap in the workflow of planning a research flight and are contributing to making research flight planning easier and more efficient.

Furthermore, we have successfully employed the OGC Web Map Service standard to provide online access to on-demand generated horizontal maps and vertical crosssections of the predictions. The WMS approach eliminates the need of transferring large amounts of model data to the campaign site while still allowing for interactivity in the visualisation. The MSS can thus be deployed at remote campaign sites with limited Internet bandwidth. The WMS standard in its latest versions 1.1.1 and 1.3.0 did not allow to implement all of our requirements in a standard-compliant way. We have hence added our own approaches to handle, for instance, vertical cross-sections.

With a case study in the scenario of the 2010 Eyjafjalla volcano eruption, we have demonstrated the applicability of our tool. The case study highlights the potential of the software to improve the flight planning process during future aircraft-based field campaigns.

We will actively use and continue to evaluate the tool during upcoming field campaigns. We also see much room for further research and developments. For instance, we recommend to investigate the control of models run in on-demand operation through web services. In this respect, it is of interest to study the applicability of other OGC web service standards to atmospheric research flight planning. This can include using the Web Processing Service ${ }^{14}$ to integrate ondemand processing of the forecast data, for instance, to compute particle trajectories on the wind field. Furthermore, we suggest to improve flight planning and flight performance computation by providing options to specify flight manoeuvres typical of research flights (e.g. downward spirals) and by automatically optimising technically infeasible flight profiles. Further research could consider investigating visualisation and data mining questions, with the objective of making the process of exploring the large amounts of prediction data that have to be handled during a campaign more efficient.

\footnotetext{
${ }^{14} \mathrm{http}: / /$ www.opengeospatial.org/standards/wps
} 
It would be interesting, for example, to automatically track features of interest to the flight planner or to visualise information about prediction uncertainty.

With the ongoing development of the WMS standard within the OGC and upcoming related developments, interoperability to third-party providers will improve in the future. This way, advanced functionality may be added to the MSS with little effort. The WMS approach will also be well suited to make new forecast and observation data accessible on-board the HALO aircraft. This will be useful to provide updated information to the aircraft crew during long-range flights.

Finally, if you are interested in the tool, please contact the authors.

\section{Supplementary material related to this article is available online at: \\ http://www.geosci-model-dev.net/5/55/2012/ gmd-5-55-2012-supplement.pdf.}

Acknowledgements. Access to ECMWF prediction data has been kindly provided in the context of the ECMWF special project "Support Tool for HALO Missions". We are very grateful to Patrick Jöckel for providing the EMAC data used in the case study and for providing helpful comments on the manuscript.

Edited by: P. Jöckel

\section{References}

Bauer, G.: Performance-Modul für das DLR/IPAMissionsplanungssystem, Diplomarbeit, Hochschule Regensburg, 2011.

Blakeslee, R., Hall, J., Goodman, M., Parker, P., Freudinger, L., and He, M.: The Real Time Mission Monitor - A Situational Awareness Tool For Managing Experiment Assets, in: NASA Science and Technology Conference 2007, 19-21 June 2007, College Park, MD, 2007.

de la Beaujardiere, J.: Web Map Service Implementation Specification, Version 1.1.1, Open Geospatial Consortium Inc., available at: http://www.opengeospatial.org/standards/wms (last access: 13 January 2012), 2002.

de la Beaujardiere, J.: OpenGIS Web Map Service Implementation Specification, Version 1.3.0, Open Geospatial Consortium Inc., available at: http://www.opengeospatial.org/standards/wms (last access: 13 January 2012), 2006.

Dörnbrack, A., Leutbecher, M., Volkert, H., and Wirth, M.: Mesoscale forecasts of stratospheric mountain waves, Met. Apps, 5, 117-126, doi:10.1017/S1350482798000802, 1998.

Eymann, G.: Web Development Activities by Central Development of DWD (FEZE), in: ECMWF Web Developers' Meeting 2010, Reading, UK, available at: http: //nwmstest.ecmwf.int/publications/member_states_meetings/ web_developers/2010/pdfs/15_DE_GE_DWD.pdf (last access: 13 January 2012), 2010.
Flatøy, F., Hov, Ø, and Schlager, H.: Chemical forecasts used for measurement flight planning during POLINAT 2, Geophys. Res. Lett., 27, 951-958, doi:10.1029/1999GL010805, 2000.

He, M., Goodman, H. M., Blakeslee, R., and Hall, J. M.: The Waypoint Planning Tool: Real Time Flight Planning for Airborne Science, in: American Geophysical Union 2010 Fall Meeting, 13-17 Dec 2010, San Francisco, Calif., 2010.

Hunter, J. D.: Matplotlib: A 2D Graphics Environment, Comput. Sci. Eng., 9, 90-95, doi:10.1109/MCSE.2007.55, 2007.

Huntrieser, H., Schlager, H., Roiger, A., Lichtenstern, M., Schumann, U., Kurz, C., Brunner, D., Schwierz, C., Richter, A., and Stohl, A.: Lightning-produced $\mathrm{NO}_{\mathrm{x}}$ over Brazil during TROCCINOX: airborne measurements in tropical and subtropical thunderstorms and the importance of mesoscale convective systems, Atmos. Chem. Phys., 7, 2987-3013, doi:10.5194/acp-7-29872007, 2007.

Jöckel, P., Kerkweg, A., Pozzer, A., Sander, R., Tost, H., Riede, H., Baumgaertner, A., Gromov, S., and Kern, B.: Development cycle 2 of the Modular Earth Submodel System (MESSy2), Geosci. Model Dev., 3, 717-752, doi:10.5194/gmd-3-717-2010, 2010.

Kertész, S., Siemen, S., Lamy-Thépaut, S., Ii, F., Russell, I., and Karhila, V.: OGC Clients in Metview 4, in: Third workshop on the use of OGC/GIS standards in meteorology, Exeter, UK, available at: http://external.opengis.org/twiki_public/pub/MetOceanDWG/ MetOGCWorkshop3/ks_ogcclient_exeter_2010.pdf (last access: 13 January 2012), 2010.

Klanner, L.: Vorhersage der Lidar-Messabdeckung zur optimierten Flugwegplanung während T-PARC, Diplomarbeit, LudwigMaximiliams-Universität München, 2009.

Konopka, P., Steinhorst, H., Grooss, J., Günther, G., Müller, R., Elkins, J. W., Jost, H., Richard, E., Schmidt, U., Toon, G., and McKenna, D. S.: Mixing and ozone loss in the 1999-2000 Arctic vortex: Simulations with the three-dimensional Chemical Lagrangian Model of the Stratosphere (CLaMS), J. Geophys. Res., 109, D02315, doi:10.1029/2003JD003792, 2004.

Lawrence, M. G., Crutzen, P. J., Rasch, P. J., Eaton, B. E., and Mahowald, N. M.: A model for studies of tropospheric photochemistry: Description, global distributions, and evaluation, J. Geophys. Res., 104, D02315, 26245-26278, doi:10.1029/1999JD900425, 1999.

Little, C.: Status of the Met Ocean Domain WG, in: Third workshop on the use of OGC/GIS standards in meteorology, Exeter, UK, available at: http://external.opengis. org/twiki_public/pub/MetOceanDWG/MetOGCWorkshop3/ OGCMetOceanDWGStatus20101115.pdf (last access: 13 January 2012), 2010.

Matula, J.: Exploiting Web Services for meteorological applications, in: Second workshop on the use of OGC/GIS standards in meteorology, Toulouse, France, available at: http://www.meteo. fr/cic/meetings/gis-ogc/presentations.html (last access: 13 January 2012), 2009.

Murray, D., McWhirter, J., Wier, S., and Emmerson, S.: The Integrated Data Viewer: a Web-enabled application for scientific analysis and visualization, in: 19th Conference on International Interactive Information and Processing Systems (IIPS) for Meteorology, Oceanography, and Hydrology, American Meteorological Society, 2003.

Murray, D., McWhirter, J., Ho, Y., and Whittaker, T. M.: IDV at 
5: New features and future, in: 25th Conference on International Interactive Information and Processing Systems (IIPS) for Meteorology, Oceanography, and Hydrology, American Meteorological Society, 2009.

Raoult, B., Bispham, P., Brady, A., Casado, J. L., Correa, R., Lamy-Thépaut, S., Orford, T., Richardson, D., Sahin, C., Siemen, S., Valiente, C., and Varela, D.: ECMWF Web re-engineering project, in: Third workshop on the use of OGC/GIS standards in meteorology, Exeter, UK, available at: http://external.opengis.org/twiki_public/pub/ MetOceanDWG/MetOGCWorkshop3/ecmwf_ogc.pdf (last access: 13 January 2012), 2010.

Roeckner, E., Brokopf, R., Esch, M., Giorgetta, M., Hagemann, S., Kornblueh, L., Manzini, E., Schlese, U., and Schulzweida, U.: Sensitivity of Simulated Climate to Horizontal and Vertical Resolution in the ECHAM5 Atmosphere Model, J. Climate, 19, 3771-3791, doi:10.1175/JCLI3824.1, 2006.

Roiger, A., Schlager, H., Schäfler, A., Huntrieser, H., Scheibe, M., Aufmhoff, H., Cooper, O. R., Sodemann, H., Stohl, A., Burkhart, J., Lazzara, M., Schiller, C., Law, K. S., and Arnold, F.: Insitu observation of Asian pollution transported into the Arctic lowermost stratosphere, Atmos. Chem. Phys., 11, 10975-10994, doi:10.5194/acp-11-10975-2011, 2011.

Russell, I., Siemen, S., Ii, F., Kertész, S., Lamy-Thépaut, S., and Karhila, V.: Metview 4 - ECMWF's latest generation meteorological workstation, ECMWF Newsletter, 126, 23-27, 2010.

Schofield, R., Frieler, K., Wohltmann, I., Rex, M., Hobe, M. V., Stroh, F., Koch, G., Peter, T., Canty, T., Salawitch, R., and Volk, C. M.: Polar stratospheric chlorine kinetics from a selfmatch flight during SOLVE-II/EUPLEX, Geophys. Res. Lett., 35, L01807, doi:10.1029/2007GL031740, 2008.

Schumann, U.: Probing the Atmosphere with Research Aircraft European Aircraft Campaings, in: Observing Systems for Atmospheric Composition, edited by: Visconti, G., DiCarlo, P., Brune, W. H., Schoeberl, M., and Wahner, A., Springer, New York, 8596, 2007.

Schumann, U., Weinzierl, B., Reitebuch, O., Schlager, H., Minikin, A., Forster, C., Baumann, R., Sailer, T., Graf, K., Mannstein, H., Voigt, C., Rahm, S., Simmet, R., Scheibe, M., Lichtenstern, M., Stock, P., Rüba, H., Schäuble, D., Tafferner, A., Rautenhaus, M., Gerz, T., Ziereis, H., Krautstrunk, M., Mallaun, C., Gayet, J.-F., Lieke, K., Kandler, K., Ebert, M., Weinbruch, S., Stohl, A., Gasteiger, J., Groß, S., Freudenthaler, V., Wiegner, M., Ansmann, A., Tesche, M., Olafsson, H., and Sturm, K.: Airborne observations of the Eyjafjalla volcano ash cloud over Europe during air space closure in April and May 2010, Atmos. Chem. Phys., 11, 2245-2279, doi:10.5194/acp-11-2245-2011, 2011.
Siemen, S., Ii, F., and Russel, I.: Metview Macro - A powerful meteorological batch language, ECMWF Newsletter, 125, 3032, 2010.

Stohl, A., Forster, C., Frank, A., Seibert, P., and Wotawa, G.: Technical note: The Lagrangian particle dispersion model FLEXPART version 6.2, Atmos. Chem. Phys., 5, 2461-2474, doi:10.5194/acp-5-2461-2005, 2005.

Summerfield, M.: Rapid GUI Programming with Python and Qt (Prentice Hall Open Source Software Development), Prentice Hall, 1st Edn., 2007.

Tosi, S.: Matplotlib for Python Developers, Packt Publishing, 2009. van der Walt, S., Colbert, S. C., and Varoquaux, G.: The NumPy Array: A Structure for Efficient Numerical Computation, Comput. Sci. Eng., 13, 22-30, doi:10.1109/MCSE.2011.37, 2011.

Voidrot-Martinez, M.-F. and Little, C.: OGC Meteorology and Oceanography Domain Working Group progress report, in: Third workshop on the use of OGC/GIS standards in meteorology, Exeter, UK, available at: http://external.opengis. org/twiki_public/pub/MetOceanDWG/MetOGCWorkshop3/ OGC_MDWG_progress_report_GIS-OGC-in-met_Nov_2010.pdf (last access: 13 January 2012), 2010.

Voigt, C., Schumann, U., Jurkat, T., Schuble, D., Schlager, H., Petzold, A., Gayet, J.-F., Krämer, M., Schneider, J., Borrmann, S., Schmale, J., Jessberger, P., Hamburger, T., Lichtenstern, M., Scheibe, M., Gourbeyre, C., Meyer, J., Kübbeler, M., Frey, W., Kalesse, H., Butler, T., Lawrence, M. G., Holzäpfel, F., Arnold, F., Wendisch, M., Döpelheuer, A., Gottschaldt, K., Baumann, R., Zöger, M., Sölch, I., Rautenhaus, M., and Dörnbrack, A.: In-situ observations of young contrails - overview and selected results from the CONCERT campaign, Atmos. Chem. Phys., 10, 90399056, doi:10.5194/acp-10-9039-2010, 2010.

Wernli, B. H. and Davies, H. C.: A lagrangian-based analysis of extratropical cyclones. I: The method and some applications, Q. J. R. Meteorol. Soc., 123, 467-489, doi:10.1002/qj.49712353811, 1997. 